\title{
The role of immunostimulants in the complex therapy of Covid 19 - a clinical case
}

\author{
Yozlem Ali Kobakova ${ }^{1}$, Maria Moneva-Sakelarieva ${ }^{1}$, Petar Atanasov ${ }^{1}$, \\ Stefka Ivanova ${ }^{2}$, Danka Obreshkova ${ }^{2,3}$ \\ 1 Clinic of Internal Diseases UMHATEM “N. I. Pirogov", Sofia, Bulgaria \\ 2 Department of Chemistry and Biochemistry, Faculty of Pharmacy, Medical University Pleven, Sofia, Bulgaria \\ 3 Faculty of Pharmacy, Medical University - Sofia, Sofia, Bulgaria \\ Corresponding author: Danka Obreshkova (phddanka@yahoo.com)
}

Received 26 August 2020 • Accepted 25 September 2020 Published 23 October 2020

Citation: Kobakova YA, Moneva-Sakelarieva M, Atanasov P, Ivanova S, Obreshkova D (2020) The role of immunostimulants in the complex therapy of Covid 19 - a clinical case. Pharmacia 67(4): 233-237. https://doi.org/10.3897/pharmacia.67.e58024

\begin{abstract}
The aim of current study was the investigation of complex treatment including nutritional supplement with recognized antioxidant and immunomodulatory properties (malic, glycyrrhizic, ascorbic and folic acids, glucosamine, arginine, glycine, calcium pantothenate, cyanocobalamine, zinc sulfate, pyridoxal) and purely plant systemic protease supplement (Serrapeptase, Bromelaim, Boswellia, Uncaria tomentosa, Quercetin) as a strategy against Covid 19. A clinical case of a 33-year-old patient treated in Internal Clinic Diseases "Pirogov" against atypical bilaterally viral pneumonia with RT-PCR proven coronavirus have been presented. A complex treatment strategy was applied: Doxycycline, Amikacin sulfate, food additive containing malic and glycyrhizic acids, aminoacids, B-vitamins, antioxidants, proteolytic agent, Methylprednisolone, anticoagulant: Nadroparin calcium, hepatoprotector: Ademethionine. After 16 days hospitalization and two negative RT-PCR tests the patient was discharged in improved condition and home treatment with Levofloxacin hemihydrate, vitamins, probiotics, immunomodulatory and proteolytic products for one month was recommended. Immunostimulating, proteolytic and other products have an important role in complex treatment.
\end{abstract}

\section{Keywords}

immunomodulators, proteolytics, pneumonia, SARS-CoV-2, treatment

\section{Introduction}

Viral infections are still a serious challenge for modern medicine, despite the constant development and improvement of the pharmaceutical industry. According to the WHO, in the last 20 years several viral epidemics have been caused by members of the genus Betacoronavirus: severe acute respiratory syndrome coronavirus (SARS$\mathrm{CoV}$ ) in 2002-2003 and middle east respiratory syndrome coronavirus (MERS-CoV), which leads to viral pneumonia and acute respiratory distress syndrome in some patients. This distress syndrome is a respiratory failure, characterized by widespread inflammation in the lungs with symptoms including shortness of breath, rapid breathing, and bluish skin coloration (Fan et al. 2018).

In December 2019, a new infectious respiratory disease was registered in Wuhan City, Hubei Province, China, with etiology, associated with a new strain of virus belonging to the family Coronaviridae. On February 11, 2020, the disease was named Covid 19. Due to the potential of this new virus to spread worldwide, on March 11, 2020, when the number of patients with Covid 19 outside China increased 13 times and the number of those affected countries tripled with the registration of the disease in more 
than 118,000 patients in 114 countries and more than 4,000 deaths, the WHO declared Covid 19 a pandemic. Till the end of August 2020 the infected people in the world reached 25,000,000 and 800,000 deaths were registered. The virus pass through the nasal and larynx mucous membranes, enters the lungs through the respiratory tract and the peripheral blood from the lungs, causing viremia (Guan et al. 2020) and then attacks the targeting organs that express angiotensin converting enzyme-2 (ACE2) receptors, present in the lungs, heart, blood vessels, kidneys and gastrointestinal tract (Zhang $\mathrm{H}$ et al. 2020).

For Covid 19 have been reported different symptoms: cough, fever, sore throat, chills, sneezing, runny nose, shortness of breath, headache, fatigue, muscle and joint pains, loss of taste, smell or appetite, nausea, vomiting, diarrhea skin lesions (Huang et al. 2020). Symptoms may appear 2-14 days after exposure to the virus, ranging from mild symptoms to severe illness (Siddiqi and Mehra 2020), especially in patient with cardiovascular diseases and after cardiac surgery (Abedinov et al. 2018).

For Covid 19, stage I is for early infection and involves an incubation period associated with mild and often non-specific symptoms: fever and a dry cough, with observed lymphopenia and neutrophilia without other significant abnormalities. During moderate stage II is developed a viral pneumonia, with cough, fever and withouth hypoxia (IIa) or with hypoxia (IIb) and with bilateral pulmonary infiltrates.(Siddiqi and Mehra 2020). In severe stage III an acute respiratory distress syndrome (Lai et al. 2020) and cytokine release syndrome (Zhang C et al. 2020) are developed. Patients have severe disease if they have any of the following criteria: respiratory rate of at least 30 breaths per min, oxygen saturation of $93 \%$ or lower in a resting state, ratio of arterial partial pressure of oxygen and oxygen concentration no greater than $300 \mathrm{~mm} \mathrm{Hg}$, or more than $50 \%$ lesion progression in lung imaging within 24-48 h (Huang et al. 2020). Very often mechanical abdominal ventilation is applied in patients with Covid 19 with acute hypoxic respiratory insufficiency like after cardiac surgery (Tsaryanski et al. 2013a; Tsaryanski et al. 2014).

For infected with Covid-19 at higher risk of developing dangerous symptoms and the quickly multi-organ failure, morbidity and mortality are people of any age with pre-existing diseases (Zhou et al. 2020):

1. lung diseases: chronic obstructive pulmonary disease, lung cancer, cystic fibrosisc, pulmonary fibrosis, moderate to severe asthma;

2. cardiovascular diseases: cardiomyopathy, high blood pressure, pulmonary hypertension, congenital heart disease, heart failure (Mehra and Ruschitzka 2020), coronary artery disease, a type of blood disorder: thalassemia, cases after cardiac surgery (Abedinov et al. 2019a; Tsaryanski et al. 2013b);

3. type 2 diabetes, severe obesity, chronic kidney or liver disease, cerebrovascular disease, dementia;

4. weakened immune system due to taking medication for an autoimmune disease, organ or bone marrow transplantation, severe immune disorders, HIV infection, cancer chemotherapy, use of corticosteroids.

Pre-existing cardiovascular diseases and cases after cardiac surgery (Abedinov et al. 2019b) are one of the very often risk factors, associated with worse outcomes and increased risk of death in patients with COVID-19 (Zheng et al. 2020), and COVID-19 can cause cardiovascular disorders: myocardial injury, arrhythmias, acute coronary syndrome, venous thromboembolism, fulminant myocarditis (Madjid et al. 2020).

The accumulated data on the development of SARS - CoV infection in 2002-2003 can be used to elucidate the mechanisms of the immune response in patients with Covid 19. In the acute phase of SARS-CoV infection, in a large number of patients (subject to monitoring and additional statistical processing) there is a rapid reduction of lymphocytes in the peripheral blood, mainly T-lymphocytes - CD 4+, CD 8+. These changes precede even changes in radiography. SARS-CoV specific antibodies are formed about two weeks after the onset of the first symptoms. During the recovery period, high and stable levels of S-protein-neutralizing specific antibodies are found in some patients - an important point in determining the outcome of the disease. During the infection, the number of white blood cells is normal or slightly reduced, lymphocytopenia is observed. It has been suggested that T-lymphocyte reduction may occur early, which may affect antibody production. Lymphocytopenia is characteristic of more severe forms of the disease and progresses with worsening of the condition ( $\mathrm{Hu}$ et al. 2020).

In pathogenesis of SARS-CoV-2 infection (Lin et al. 2020) in severe stage III a systemic hyperinflammation is developed by overproduction by the immune system (Shi et al. 2020) of significant elevated pro-inflammatory cytokines and chemokines: interleukin $1 \beta$, interveukin 2 , interveukin 6 , interveukin 7 , tumor necrosis factor- $\alpha$, granulocyte colony-stimulating factor, macrophage inflammatory protein 1- $\alpha, C$-reactive protein resulting in cytokine storm (Moore and June 2020). The multiorgan damage from Covid 19 infection is caused by hyperinflammation, hypercoagulability, thromboinflammation and thromboembolis (Connors and Levy 2020). In Covid 19 infection a decrease in helper, suppressor, and regulatory T cell is observed (Siddiqi et al. 2020).

No drug or vaccine has yet been approved for treayment of Covid 19. Despite ongoing efforts to establish etiological treatments, therapeutic strategies to control infection are currently limited to the use of symptomatic and supportive drugs, prevention of superimposed bacterial infections (Azithromycin), inflammation control (Dexamethasone), restorative compounds, oligonucleotide-based therapies (Sanders et al. 2020).

The global innovative pharmaceutical industry has accelerated its efforts to search for application of known medicinal products with a known antiviral effect and to develop new antiviral drugs. About 30 antiviral drugs at various stages of development or use are currently being 
studied by innovative pharmaceutical companies to determine their effectivity agaunst Covid-19. The efferds are related for acceleration of the development of therapeutic tools to deal with current and future coronavirus outbreaks. Clinical trials for investigation of the effectiveness of HIV/AIDS drugs and of antiviral combinations: Daclatasvir/Sofosbuvir, Lopinavir/Ritonavir, nucleoside analogues (Favipiravir, Galidesivir, Remdesivir, Ribavirin) have been launched. Oseltamivir, Stopcivir and Umifenovir are under clinical investigations too ( $\mathrm{Li}$ and De Clercq 2020). Ribavirin, Remdesivir, Galidesivir, Sofosbuvir and Tenofovir show promising results for application against Covid 19 (Elfiky 2020).

In-depth immunological studies are needed to understand the pathogenesis, improve the treatment of the disease and improve the prognosis. More and more attention is being paid to the role of immunomodulatory drugs: monoclonal antibodies, vaccines, convalescent plasma, Apilimod - against autoimmune diseases, herbal immunomodulators, recombinant Bacillus Calmette-Guérin (BCG) vaccine VPM1002 (Sanders et al. 2020).

Against COVID-19 ate being investigated 3 types of vaccines (Lurie et al. 2020): inactive whole virus vaccines, nucleic acid vaccines and subunit vaccines, which prevent the docking of the S-spike protein with the host ACE2 receptors (Chen et al. 2020).

Through scientific knowledge in the field of infectious diseases and antibody detection technology, rapidly are mobilized research efforts for clinical trials for potential coronavirus neutralizing monoclonal antibodies (Acalabrutinib, Avdoralimab, Mavrilimumab, Ravulizumab, Ruxolitinib, Sarilumab, Siltuximab). For the treatment and prevention of Covid-19 very important clinical evaluation are going for Tocilizumab - a human monoclonal antibody that inhibits the interleukin-6 pathway by binding to and blocking the IL- 6 receptor (Zhang $\mathrm{C}$ et al. 2020). A very few very special SARS-CoV-2 cross-neutralizing antibodies have been documented, including ACE2 nonblockers: 47D11 and S309, and ACE2 blocker VHH-72 (Ho 2020).

All these data should draw the attention of clinicians to the inclusion of drugs with immunostimulatory, general strengthening and restorative action in therapeutic regimens. The aim of current study is the investigation of the contribution of complex therapy to a good outcome of the Covid 19 disease and the important role of agents with immunostimulating and proteolytic action such as malic acid, glycyrrhizic acid, glucosamine, arginine, glycine, calcium pantothenate, ascorbic acid, folic acid, cyanocobalamine, zinc sulfate, pyridoxal and Serrapeptase, Bromelaim, Boswellia, Uncaria tomentosa, Quercetin

\section{Clinical case: methods}

A clinical case of a 33-year-old patient treated in the Internal Medicine Clinic of NI Pirogov University Hospital, Sofia, on the occasion of viral pneumonia with RT-PCR of proven coronavirus etiology has been presented. Laboratory tests, radiography and computed tomography were used.

\section{Results and discussion}

The patient was with complaints of cough, sore throat and fever of about $38^{\circ} \mathrm{C}$ for $4-5$ days, treated with Amoxiclav at home on the instructions of a personal doctor and was without past and concomitant diseases and withouth history of smoking. The objective condition on admission were: severely damaged general condition, asthenic habit, pale cyanotic, intoxicated skin and visible mucous membranes, subfebrile at the time of examination $37.7^{\circ} \mathrm{C}$, with not enlarged peripheral lymph nodes in accessible places and no abnormalities in neurological status.

In respiratory system was observed: normosthenic thorax, preserved respiratory motility, sonorous percutaneous tone, shortened bilateral basal, bilateral vesicular respiration weakened in the middle lung fields and pulmonary bases, with added small moist non-ringing rales, subcapular and atmospheric air saturation $88 \%$. For cardiovascular system was observed: rhythmic heart activity, sinus tachycardia, heart rate 110 beats / min, RR - 100/60, physiological peristalsis.

The patient had a negative rapid test for SARS-CoV 2 and a positive RT-PCR. From the conducted laboratory tests were found reduced number of leukocytes, dyselectrolytemia, increased values of markers of inflammation - CRP four times above normal, high values of transaminases, coagulation disorders - increased values of $\mathrm{D}$-dimer and fibrinogen; low saturation requiring oxygen therapy.

From the imaging studies - radiography were demonstrated the following: in the right axillary transparency of the parenchyma was reduced as type of frosted glass (in the clinic possible inflammatory changes of atypical genesis), not lightened bone-diaphragmatic sinuses, mediastinal shadow located in the middle and S-shaped scoliosis.

In computed tomography examination of the lung on the first day of hospitalization were observed no evidences for compression or dislocation of trachea and main bronchi and for effusions in pleural spaces, no data for enlarged axillary lymph nodes bilaterally and for enlarged (over $10 \mathrm{~mm}$ ) mediastinal lymph nodes. By computed tomography for lungs were observed: the presence of zones of consolidation of the parenchyma of the type of atypical pneumonia with viral origin bilaterally, most pronounced on the right axillary and left basal with a tendency to confluence on the type of mosaic reconstruction (crazy paving). From the conducted clinical and laboratory examinations the patient was diagnosed with atypical pneumonia with coronavirus genesis.

The following treatment regimen was applied:

1. combination antibiotic therapy in optimal doses Doxycycline initial dose $200 \mathrm{mg}$ once, followed by 100 mg daily, Amikacin sulfate 500 mg twice daily; 
2. vitamins - ascorbic acid - 1 g daily; thiamin nitrate, riboflavin, niacin, pantothenic acid, pyridoxine hydrochloride, biotin, folic acid, cobalamine - twice daily intravenously;

3. glucocorticoid - Methylprednisolone $40 \mathrm{mg}$ daily;

4. stress ulcer prevention - Famotidine;

5. infusions of aqueous saline solutions;

6. anticoagulants - Nadroparin calcium;

7. hepatoprotector - Ademethionine;

8. orally applicable essential oils;

9. oxygen therapy;

10. antiviral and immunostimulating product - malic acid, glycyrrhizic acid, glucosamine, arginine, glycine, calcium pantothenate, ascorbic acid, folic acid, cyanocobalamine, zinc sulfate, pyridoxal;

11. proteolytic product - Serrapeptase, Bromelaim, Boswellia, Uncaria tomentosa, Quercetin.

During the hospital stay were observed gradual improvement in the general condition: reduction of shortness of breath, prolongation of afebrile periods and increase of physical capacity. After the $4^{\text {th }}$ day the patient was without the need for supplemental oxygen therapy, with good saturation of atmospheric air, reduced cough. From computed tomography examination of the lung on the $10^{\text {th }}$ day of hospitalization were observed no evidences for compression or dislocation of trachea and main bronchi and no data for pleural and pericardial effusion, not detected significantly large (over $10 \mathrm{~mm}$ ) mediastinal lymph nodes and no data for enlarged axillary lymph nodes bilaterally.

On the $10^{\text {th }}$ day the described changes of frosted glass type in the lung parenchyma of the previous computed tomography examination persisted in a slightly increased volume and with reduced density. The data for changes in the lung parenchyma by type of atypical pneumonia with viral origin were confirmed.The hospital treatment continued for another 6 days, with the following change in the therapeutic regimen: discontinuation of oxygen therapy and continuation with immunomodulatory product (malic acid, glycyrrhizic acid, glucosamine, arginine, glycine, calcium pantothenate, ascorbic acid, folic acid, cyanocobalamine, zinc sulfate, pyridoxal) and proteolytic product (Serrapeptase, Bromelaim, Boswellia, Uncaria tomentosa, Quercetin) - 3 times 1 powder a day.

In two consecutive negative RT-PCR tests, the patient was discharged after 16 days of hospital treatment in a significantly improved general condition - permanently afebrile, hemodynamically stable, without shortness of breath and cough. The home recommended treatment was with Levofloxacin hemihydrate, vitamins, probiotics and guidelines for immunomodulatory product and proteolytic product for one month. In view of the persistent computed tomography finding of the lung, the patient was referred for a control imaging examination after the expiration of the 28-day quarantine.

After 36 days of dehospitalization, in the control examination according to the recorded anamnesis - pa- tient was without reduction of physical capacity and without deviations physical status. From examination of respiratory system were confirmed: pure vesicular respiration, without added wheezing, 99\% saturation of atmospheric air, blood indicators were in norm, with correction of the deviations in the laboratory indicators registered during hospitalization.

From computed tomography examination of the lung after 36 days of dehospitalization the were found no data for compression or dislocation of trachea and main bronchi and for enlarged axillary lymph nodes bilaterally, no evidences for pleural and pericardial effusion, mediastinum was without pathological formations and no significant lymphadenopathy and no lesions in bone structures was confirmed. After 36 days of dehospitalization the lung was with excellent resorption results: aerated parenchyma without pathological changes. Pleurodiaphragmatic adhesions bilaterally and pronounced scoliotic deformity in the distal cervical and thoracic spine were detected. The patient was instructed to continue taking proteolytic agent for another month.

\section{Conclusion}

The presented clinical case shows that young active people without concomitant diseases can be severely affected by SARS-CoV-2 infection and their severe general condition can be an indication for timely hospitalization. A multidisciplinary approach is necessary for timely diagnosis and adequate assessment of the severity of the disease. Individually targeted therapy, including drugs with restorative and restorative action and adjusted in a timely manner according to the dynamics of the patient's condition, is the key to a good outcome of the disease. In the fight against this new and unknown virus, the main steps must be the precise diagnosis, the complex therapy, the optimal hospital stay, as well as the adequate follow-up of the patient after discharge.

Each of the components of the therapy contributes to a good outcome of the disease. It is important that agents with immunostimulating (malic acid, glycyrrhizic acid, glucosamine, arginine, glycine, calcium pantothenate, ascorbic acid, folic acid, cyanocobalamine, zinc sulfate, pyridoxal) and proteolytic action (Serrapeptase, Bromelaim, Boswellia, Uncaria tomentosa, Quercetin) are actively used by clinicians and have an important role in complex treatment for good outcome of Covid 19 disease.

\section{Acknowledgements}

Authors thank the patient for his cooperation in the rehabilitation process and for agreement for publication of the results. 


\section{References}

Abedinov F, Krastev P, Petrov I, Iliev R, Tsvetanova K (2018) Risk factors for prolonged length of stay in the ICU following cardiac surgery. International Journal of Science and Re-search 7(3): 67-71.

Abedinov F, Bakalova N, Krastev P, Petrov I, Marinova R, Tzatyanski G (2019a) Survival and quality of life of patients with a prolonged stay in the intensive care unit after cardiac surgeries - remote results. Comptes rendus de l'Académie bulgare des Sciences 72(8): 1130-1136 https://doi.org/10.7546/CRABS.2019.08.16

Abedinov F, Krastev P, Marinova R, Petrov I, Bakalova N, Iliev R, Angelov H (2019b) Perioperative factors linked to prolonged length of stay in the ICU following cardiac surgery - analysis of distant results in survivors. Comptes rendus de l'Académie bulgare des Sciences 72(12): 1697-1705. https://doi.org/10.7546/CRABS.2019.12.14

Chen WH, Strych U, Hotez PJ, Bottazzi ME (2020) The SARS-CoV-2 vaccine pipeline: an overview. Current Tropical Medicine Reports 7(2): 61-64. https://doi.org/10.1007/s40475-020-00201-6

Conors JM, Levy JH (2020) Thromboinflammation and the hypercoagulability of COVID-19. Journal of Thrombosis and Haemostasis 18(7) 1559-1561. https://doi.org/10.1111/jth.14849

Elfiky AA (2020) Ribavirin, Remdesivir, Sofosbuvir, Galidesivir, and Tenofovir against SARS-CoV-2 RNA dependent RNA polymerase (RdRp): a molecular docking study. Life Science 253: 1-6. https:// doi.org/10.1016/j.lfs.2020.117592

Fan E, Brodie D, Slutsky AS (2018) Acute respiratory distress syndrome: advances in diagnosis and treatment. Journal of the American Medical Association 319(7): 698-710. https://doi.org/10.1001/jama.2017.21907

Guan W, Ni Z, Hu Y, Liang W, Ou C, He J, Liu L, Shan H, Lei C, Hui DSC, Du B, Li L, Zeng G, Yuen K-Y, Chen R, Tang C, Wang T, Chen P, Xiang J, Li S, Wang J-L, Liang Z, Peng Y, Wei L, Liu Y, Hu Y-H, Peng P, Wang J-M, Liu J, Chen Z, Li G, Zheng Z, Qiu S, Luo J, Ye C, Zhu S, Zhong N (2020) Clinical characteristics of Coronavirus disease 2019 in China. New England Journal of Medicine 382(18): 1708-1720. https://doi.org/10.1056/NEJMoa2002032

Ho M (2020) Perspectives on the development of neutralizing antibodies against SARS-CoV-2. Antibody Therapeutics 3(2): 109-114. https:// doi.org/10.1093/abt/tbaa009

Hu D, Li L, Shi W, Zhang L (2020) Less expression of CD $4^{+}$and CD $8^{+} \mathrm{T}$ cells might reflect the severity of infection and predict worse prognosis in patients with COVID-19: Evidence from a pooled analysis. Clinica Chimica Acta 510: 1-4. https://doi.org/10.1016/j.cca.2020.06.040

Huang C, Wang Y, Li X, Ren L, Zhao J, Hu Y, Zhang L, Fan G, Xu J, Gu X, Cheng Z, Yu T, Xia J, Wei Y, Wu W, Xie X, Yin W, Li H, Liu M, Xiao Y, Gao H, Guo L, Xie J, Wang G, Jiang, Gao Z, Jin Q, Wang J, Cao B (2020) Clinical features of patients infected with 2019 novel coronavirus in Wuhan, China. Lancet 395(10223): 497-506. https:// doi.org/10.1016/S0140-6736(20)30183-5

Lai CC, Shih TP, Ko WC, Tang HJ, Hsueh PR (2020) Severe acute respiratory syndrome coronavirus 2 (SARS-CoV-2) and coronavirus disease-2019 (COVID-19): the epidemic and the challenges. International Journal of Antimicrobial Agents 55(3): 1-10. https://doi. org/10.1016/j.ijantimicag.2020.105924

Li G, De Clercq E (2020) Therapeutic options for the 2019 novel coronavirus (2019-nCoV). Nature Reviews Drug Discovery 19(3): 149-150. https://doi.org/10.1038/d41573-020-00016-0

Lin L, Lu L, Cao W, Li T (2020) Hypothesis for potential pathogenesis of SARS-CoV-2 infection-a review of immune changes in patients with viral pneumonia. Emerging Microbes Infections 9(1): 727-732. https://doi.org/10.1080/22221751.2020.1746199

Lurie N, Saville M, Hatchett R, Halton J (2020) Developing COVID-19 vaccines at pandemic speed. New England Journal of Medicine 382(21): 1969-1973. https://doi.org/10.1056/NEJMp2005630

Madjid M, Safavi-Naeini P, Solomon SD, Vardeny O (2020) Potential effects of coronaviruses on the cardiovascular system: a review. Journal of the American Medical Association Cardiology 5(7): 831-840. https://doi.org/10.1001/jamacardio.2020.1286

Mehra MR, Ruschitzka F (2020) COVID-19 illness and heart failure. Journal of the Ame-rican College of Cardiology: Heart Failure 8(6): 512-514. https://doi.org/10.1016/j.jchf.2020.03.004

Moore JB, June CH (2020) Cytokine release syndrome in severe COVID-19. Science 368(6490): 473-474. https://doi.org/10.1126/science.abb8925

Sanders JM, Monogue ML, Jodlowski TZ, Cutrell JB (2020) Pharmacologic treatments for coronavirus disease 2019 (COVID-19): a review. Journal of the American Medical Association 323(18): 1824-1836. https://doi.org/10.1001/jama.2020.6019

Shi Y, Wang Y, Shao C, Huang J, Gan J, Huang X, Bucci E, Piacentini M, Ippolito G, Melino G (2020) COVID-19 infection: the perspectives on immune responses. Cell Death Differentiation 27(5): 1451-1454. https://doi.org/10.1038/s41418-020-0530-3

Siddiqi HK, Mehra MR (2020) Covid-19 illness in native and immunosuppressed states: a clinical-therapeutic staging proposal. Journal of Heart and Lung Transplantation 39(5): 405-407. https://doi. org/10.1016/j.healun.2020.03.012

Tsaryanski G, Abedinov F, Cholakov V, Markovski Al, Tenev B, Manolova V, Nachev G (2013a) Mechanical abdominal ventilation in patients with acute hypoxic respiratory insufficiency after cardiac surgery. Anesthesiology and Internal Therapy 42(3): 7-10.

Tsaryanski G, Cholakov V, Abedinov F, Tenev B, Dimitrova V, Manolova V, Natsev I, Nachev G (2013b) Risk assessment, postoperative morbidity and prognosis in patients after cardiac surgery based on the EuroSCORE and SOFA systems. Anesthesiology and Internal Therapy 42(1): 26-29.

Tsaryanski G, Abedinov F, Cholakov V, Tenev B, Panayotova S, Manolova V, Natsev I, Nachev G (2014) Application of non-invasive ventilation after planned extubation in cardiac surgical patients: frequency of reintubation. Anesthesiology and Internal Therapy 43(1): 10-12.

Zhang C, Wu Z, Li JW, Zhao H, Wang GQ (2020) The cytokine release syndrome (CRS) of severe COVID-19 and Interleukin-6 receptor (IL-6R) antagonist Tocilizumab may be the key to reduce the mortality. International Journal of Antimicrobial Agents 55(5): 1-6. https:// doi.org/10.1016/j.ijantimicag.2020.105954

Zhang H, Penninger JM, Li Y, Zhong N, Slutsky AS (2020) Angiotensin-converting enzyme 2 (ACE2) as a SARS-CoV-2 receptor: molecular mechanisms and potential therapeutic target. Intensive Care Medicine 46(4): 586-590. https://doi.org/10.1007/s00134-020-05985-9

Zheng Y-Y, Ma Y-T, Zhang J-Y, Xie X (2020) COVID-19 and the cardiovascular system. Nature Reviews Cardiology 17(5): 259-260. https:// doi.org/10.1038/s41569-020-0360-5

Zhou F, Yu T, Du R, Fan G, Liu Y, Liu Z, Xiang J, Wang Y, Song B, Gu X, Guan L, Wei Y, Li H, Wu X, Xu J, Tu S, Zhang Y, Chen H, Bin B (2020) Clinical course and risk factors for mortality of adult inpatients with COVID-19 in Wuhan, China: a retrospective cohort study. Lancet 395(10229): 1054-1062. https://doi.org/10.1016/ S0140-6736(20)30566-3 